\title{
Metamizole-Induced Type I Kounis Syndrome
}

\author{
Catarina Lameiras, Ana Corte-Real, Ana Órfão, Marta Mendes Lopes, Maria do Céu Dória
}

Department of Internal Medicine, Hospital Professor Doutor Fernando Fonseca EPE, Amadora, Portugal

Received: $26 / 12 / 2020$

Accepted: 02/01/2021

Published: 08/02/2021

How to cite this article: Lameiras C, Corte-Real A, Órfão A; Mendes Lopes M, do Céu Dória M. Metamizole-induced type I Kounis syndrome. EJCRIM 2021;8: doi:10.12890/2021_002249.

Conflicts of Interests: The Authors declare that there are no competing interests.

This article is licensed under a Commons Attribution Non-Commercial 4.0 License

\section{ABSTRACT}

Kounis syndrome (KS) is defined as acute coronary syndrome (ACS) triggered by mast cell and platelet activation in the setting of allergic or anaphylactic insults. KS is a unique and complex cause of ACS and many cases may be missed due to its highly variable clinical manifestations. In this report, we present a case of KS type I triggered by metamizole in the absence of a previous history of allergy to this drug. Following the administration of metamizole, the patient developed generalized acute urticaria, chest pain and diaphoresis. Electrocardiography (ECG) showed ST-segment elevation suggestive of myocardial infarction complicated by ventricular tachycardia. No coronary disease was observed on coronary angiography. The cardiac manifestations of KS may be life-threatening, and so it is important to appropriately recognize and treat this condition.

\section{LEARNING POINTS}

- Kounis syndrome (KS) diagnosis requires a high index of suspicion and should be considered in patients who present with acute coronary syndrome (ACS) soon after the administration of a new medication or possible allergic stimulus.

- Treatment should be administered carefully, since some drugs used to treat the cardiac manifestations of KS can worsen the allergic reaction.

- The prognosis is generally good with appropriate treatment, but some complications may occur, such as malignant arrhythmia.

\section{KEYWORDS}

Kounis syndrome, anaphylactic reaction, myocardial infarction, metamizole

\section{CASE DESCRIPTION}

A 62-year-old man was admitted to the emergency department with acute pancreatitis. His medical history included alcohol and tobacco consumption and allergy to penicillin manifested by urticaria.

During the administration of $2 \mathrm{~g}$ of intravenous (IV) metamizole for pain relief, the patient presented chest pain, diaphoresis, generalized acute urticaria and tachycardia (pulse rate of 151 beats, blood pressure of 116/66 mmHg).

The ECG showed ST-elevation in DII-DIII-aVF alternating with non-sustained monomorphic ventricular tachycardia with a pulse (Fig. 1). Echocardiography revealed a normal left ventricular ejection fraction, normal right heart chambers and no contractility abnormalities. Blood tests revealed elevated levels of myoglobin ( $139 \mathrm{ng} / \mathrm{ml}$; normal < $96 \mathrm{ng} / \mathrm{ml}$ ) and troponin I ( $1.73 \mathrm{ng} / \mathrm{ml}$; normal <0.04 ng/ml).

The patient was treated with IV hydrocortisone $200 \mathrm{mg}$ and clemastine $2 \mathrm{mg}$ for the allergic reaction. Morphine $2 \mathrm{mg}$, metoclopramide 10 $\mathrm{mg}$, acetylsalicylic acid $300 \mathrm{mg}$, clopidogrel $300 \mathrm{mg}$ and low-molecular-weight heparin $1 \mathrm{mg} / \mathrm{kg}$ were given for the acute treatment of ACS. IV amiodarone $300 \mathrm{mg}$ was administered for cardiac rhythm and rate control. Emergency coronary angiography did not show significant coronary lesions. 

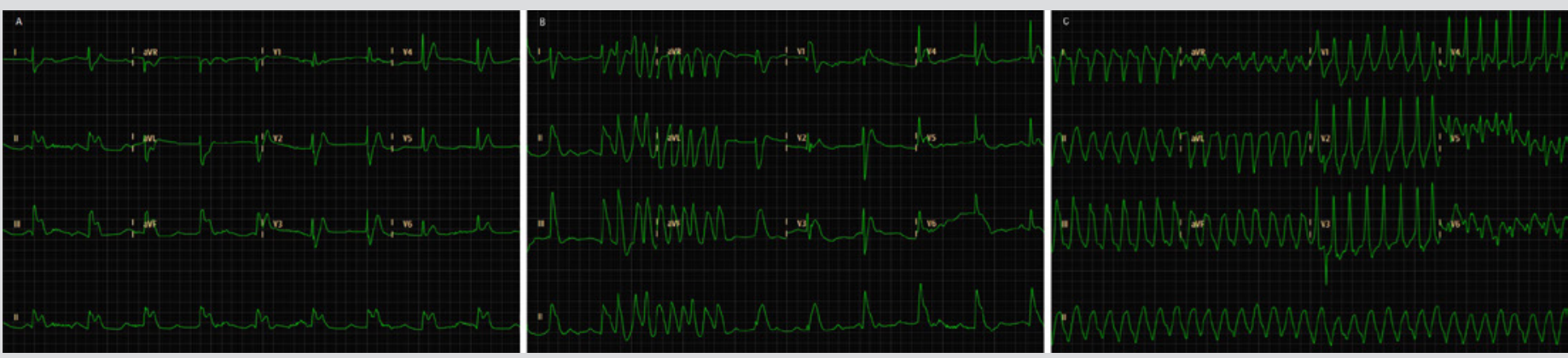

Figure 1. ECG on admission displaying a normal sinus rhythm with an acute inferior-lateral myocardial ischaemia pattern, and ST elevation in DII-DIII-aVF (A), alternating with monomorphic ventricular tachycardia (B and C)

Based on the coincidental occurrence of an allergic reaction and ACS with ST elevation in the absence of coronary disease on angiography, the patient was diagnosed with metamizole-induced KS type I.

After the diagnosis of KS was established and coronary heart disease was excluded, the patient was medicated with diltiazem 120 mg per day with remission of angina and normalization of cardiac markers. ECG revaluation on the 4th day showed sinus rhythm, pathological Q waves in DIII, T wave inversion in DII-DIII-aVF and normalization of the ST elevation (Fig. 2). Pre-discharge echocardiography revealed completely normal contractility. The patient was discharged on the 5th day and referred to the Allergy Unit.

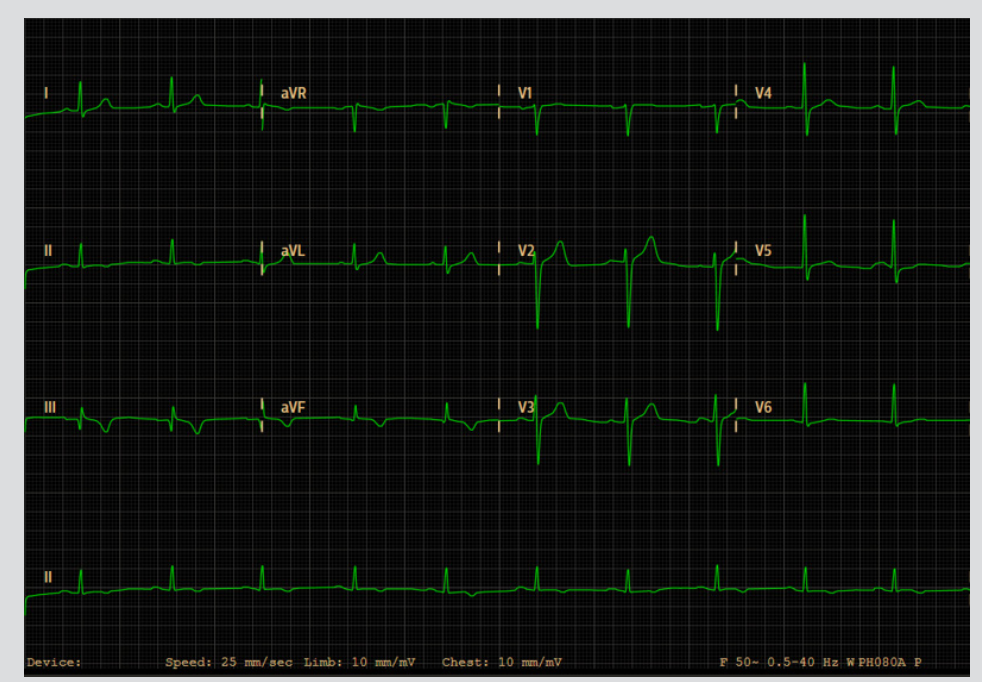

Figure 2. ECG revaluation on the 4th day after admission displaying sinus rhythm, pathological Q waves in DIII, T-wave inversion in DII-DIII-aVF and normalization of the ST elevation

\section{DISCUSSION}

KS was first described in 1991 as an 'allergic angina syndrome'[1]. Since the first reports, a common pathway for allergic and non-allergic coronary events has been demonstrated, based on the existence in allergic coronary events of a much higher degree of mast cell degranulation at plaque erosion or rupture sites compared with that in post-acute myocardial infarction of non-allergic aetiology [1]. Currently, KS is defined as the concurrence of ACS associated with mast cell and platelet activation in the setting of hypersensitivity and allergic or anaphylactic insults ${ }^{[1]}$.

Determining its true incidence is difficult, since many cases may be missed or underdiagnosed due to its variable manifestations and physician unawareness ${ }^{[1]}$.

Risk factors include a history of previous allergy and atherosclerosis ${ }^{[2]}$. Various triggers have been reported, including environmental exposures (e.g., insect bites, food) and common drugs (e.g., aspirin, antihypertensives, antibiotics, non-steroidal anti-inflammatory drugs) ${ }^{[1,2]}$. We found very few published case reports of metamizole-induced KS. The safety of metamizole has been the subject of considerable controversy due to its rare but life-threatening side effects such as anaphylactic shock and agranulocytosis, and so it has been banned in 
many countries. However, it is still available in some countries to treat certain kinds of pain ${ }^{[3]}$. Most importantly, one should balance the benefits and harms of any drug before its administration as well as past history of hypersensitivity to similar drugs.

KS pathophysiology involves coronary artery spasm and/or atheromatous plaque erosion or rupture. If anaphylaxis occurs, pre-existing coronary artery disease is considered a risk factor ${ }^{[4]}$. KS is classified into three subtypes according to the condition of the coronary arteries. Type I is characterized by coronary spasm in normal arteries which induces either ACS with normal cardiac enzymes or which may progress to acute myocardial infarction with raised cardiac enzymes ${ }^{[4]}$. Type II is characterized by coronary spasm together with plaque erosion or rupture in a patient with angiographic evidence of coronary artery disease, and type III includes patients already treated with stenting for coronary thrombosis in which the allergic reaction causes stent thrombosis ${ }^{[5]}$.

The case herein reported was compatible with KS type I, since coronary angiography did not show coronary disease. Considering the patient's age and smoking habit, ST-segment change and cardiac enzyme elevation probably occurred due to temporary vasospasm in arteries with some degree of microvascular coronary disease not detectable by angiography.

The diagnosis of KS is based on clinical signs and symptoms, along with laboratory, ECG, echocardiographic and angiographic evidence. A careful review of medication and allergies is imperative ${ }^{[1,2]}$. The clinical spectrum is highly variable and depends on the initial allergic response, the patient's comorbidities and level of exposure to the allergen ${ }^{[5]}$. Cardiac symptoms include malaise, chest pain with anginal characteristics, or palpitations. Simultaneously, typical cutaneous, respiratory and digestive anaphylactic manifestations may occur ${ }^{[1,2]}$. Laboratory work-up includes investigation of myocardial injury enzymes. ECG usually shows ST-T changes suggestive of ischaemia and echocardiography can show regional wall motion abnormalities in the distribution of the affected artery. Coronary angiography may reveal vasospasm or stenosis ${ }^{[2]}$.

Therapeutic management of KS is challenging, involving treatment of the allergic reaction and myocardial revascularization ${ }^{[4]}$. Treatment should be performed carefully, since drugs administered to treat cardiac manifestations can worsen allergy, and the drugs given for the allergic reaction can aggravate the cardiac dysfunction ${ }^{[2]}$. For example, beta-blockers and morphine should be avoided as they may trigger the release of histamine ${ }^{[5]}$.

There are no specific guidelines for the treatment of KS and most of the evidence is based on case reports ${ }^{[4]}$. In patients with the type I variant, treatment of the allergic reaction can resolve the cardiac manifestations by itself. Medical treatment should include hydrocortisone and IV H1 or $\mathrm{H} 2$ antihistamines ${ }^{[4,5]}$. Vasodilators such as calcium channel blockers and nitrates can resolve vasospasm ${ }^{[1]}$. In type II and type III KS, treatment includes myocardial revascularization based on the ACS guidelines together with allergic symptom management ${ }^{[1]}$.

Complete recovery is expected with appropriate treatment, however rare complications may occur ${ }^{[2]}$. Our patient manifested periods of ventricular tachycardia as a complication of KS. Since a good prognosis depends on proper and immediate diagnosis and rapid work-up, medical professionals should be aware of this condition.

\section{REFERENCES}

1. Kounis NG. Kounis syndrome: an update on epidemiology, pathogenesis, diagnosis and therapeutic management. Clin Chem Lab Med 2016;54(10):1545-1559.

2. Abdelghany M, Subedi R, Shah S, Kozman H. Kounis syndrome: a review article on epidemiology, diagnostic findings, management and complications of allergic acute coronary syndrome. Int J Cardiol 2017;232:1-4.

3. Kötter T, da Costa BR, Fässler M, Blozik E, Linde K, Jüni P, et al. Metamizole-associated adverse events: a systematic review and metaanalysis. PLoS One 2015;10(4): e0122918.

4. Brown SG. Cardiovascular aspects of anaphylaxis: implications for treatment and diagnosis. Curr Opin Allergy Clin Immunol 2005;5(4):359-364.

5. Fassio F, Losappio L, Antolin-Amerigo D, et al. Kounis syndrome: a concise review with focus on management. Eur J Intern Med 2016;30:7-10. 